\title{
CAPACITY AND MEASURE DENSITIES
}

\author{
O. MARTIO
}

\section{Introduction}

Let $h:[0, \infty) \rightarrow[0, \infty)$ be a measure function, i.e. $h$ is continuous, strictly increasing, $h(0)=0$, and $\lim _{t \rightarrow \infty} h(t)=\infty$, and let

$$
H_{h}(A)=\inf \left\{\sum_{i} h\left(r_{i}\right): \bigcup_{i} \bar{B}^{n}\left(x_{i}, r_{i}\right) \supset A\right\}
$$

be the $h$-(outer)measure of $A \subset R^{n}$. The upper $h$-measure density of $A$ at $x \in R^{n}$ is

$$
\Theta_{h}(x, A)=\varlimsup_{r \rightarrow 0} H_{h}\left(A \cap \bar{B}^{n}(x, r)\right) / h(r) .
$$

Assume that $1<p \leqq n$ and that $C$ is a closed set in $R^{n}$. For $x \in R^{n}$

$$
\operatorname{cap}_{p}(x, C)=\varlimsup_{r \rightarrow 0} r^{p-n} \operatorname{cap}_{p}\left(B^{n}(x, 2 r), \bar{B}^{n}(x, r) \cap C\right)
$$

defines the upper $p$-capacity density of $C$ at $x$. Here cap $p$ on the right hand side is the ordinary variational $p$-capacity of a condenser.

The purpose of this note is to compare $\Theta_{h}(x, C)$ and $\operatorname{cap}_{p}(x, C)$ for various $h$ and $p$. Among other things we show that $\operatorname{cap}_{p}(x, C)=0$ implies $\Theta_{h}(x, C)=0$ for $h(r)=r^{\alpha}$, where $\alpha>n-p$. As a byproduct some measure theoretic properties of sets $C$ which satisfy $\operatorname{cap}_{p}(x, C)=0$ for all $x \in C$ are given. Observe that such a set $C$ need not be of zero $p$-capacity.

We shall mainly employ the method due to Ju. G. Rešetnjak, cf. [7, 8]. There is an extensive literature on measure theoretic properties of sets of zero $p$-capacity, see e.g. [1], [6], [7, 8], and [10].

\section{Preliminary results}

2.1. Notation. The open ball centered at $x \in R^{n}$ with radius $r>0$ is denoted by $B^{n}(x, r)$. We abbreviate $B^{n}(r)=B^{n}(0, r)$ and $S^{n-1}(r)=\partial B^{n}(r)$. The Lebesgue measure in $R^{n}$ is denoted by $m$ and $\Omega_{n}=m\left(B^{n}(1)\right)$. We let $\omega_{n-1}$ denote the $(n-1)-$ measure of $S^{n-1}(1)$. For $p \geqq 1, L^{p}$ is the class of all $p$-integrable functions in $R^{n}$ with the norm \|\|$_{p}$. 
If $A \subset R^{n}$ is open, then $C_{0}^{1}(A)$ means the set of continuously differentiable real valued functions with compact support in $A$. For $u \in C_{0}^{1}(A), \nabla u=\left(\partial_{1} u, \ldots, \partial_{n} u\right)$ is the gradient of $u$. Each $u$ has the representation, cf. [7, Lemma 3],

$$
u(x)=\frac{1}{\omega_{n-1}} \int_{R^{n}} \frac{\nabla u(y) \cdot(x-y)}{|x-y|^{n}} d m(y) .
$$

If $A$ is open in $R^{n}$ and $C \subset A$ is compact, then the pair $(A, C)$ is called a condenser and its $p$-capacity, $1<p \leqq n$, is defined by

$$
\operatorname{cap}_{p}(A, C)=\inf _{u \in W(A, C)} \int_{A}|\nabla u|^{p} d m
$$

where $W(A, C)$ is the set of all non-negative functions $u \in C_{0}^{1}(A)$ with $u(x)>1$ for all $x \in C$. Note that for $x \in R^{n}$ and $0<r_{1}<r_{2}$

$$
\operatorname{cap}_{p}\left(B^{n}\left(x, r_{2}\right), \bar{B}^{n}\left(x, r_{1}\right)\right)= \begin{cases}\omega_{n-1}\left(\left(r_{2}^{q}-r_{1}^{q}\right) / q\right)^{1-p}, & p \in(1, n) \\ \omega_{n-1}\left(\ln \left(r_{2} / r_{1}\right)\right)^{1-n}, & p=n,\end{cases}
$$

where $q=(p-n) /(p-1)$. The following subadditivity result for capacities is wellknown:

2.4. Lemma. Suppose that $(A, C)$ is a condenser. If $\left(A_{i}, C_{i}\right), i=1,2, \ldots$, is a sequence of condensers such that $A \supset A_{i}$ and $\cup C_{i} \supset C$, then

$$
\operatorname{cap}_{p}(A, C) \leqq \sum \operatorname{cap}_{p}\left(A_{i}, C_{i}\right)
$$

If $C$ is closed in $R^{n}, x \in R^{n}$, and $r>0$ we let

$$
\operatorname{cap}_{p}(x, C, r)=r^{p-n} \operatorname{cap}_{p}\left(B^{n}(x, 2 r), \bar{B}^{n}(x, r) \cap C\right) .
$$

The set $C$ is of zero $p$-capacity, abbreviated $\operatorname{cap}_{p} C=0$, if for all compact sets $C^{\prime} \subset C, \operatorname{cap}_{p}\left(A, C^{\prime}\right)=0$ for all open $A \supset C^{\prime}$.

If $h$ is a measure function, then in addition to the measure $H_{h}$ defined in the introduction we use the $h$-Hausdorff measure

$$
H_{h}^{*}(A)=\liminf _{t \rightarrow 0}\left\{\sum h\left(r_{i}\right): \cup \bar{B}^{n}\left(x_{i}, r_{i}\right) \supset A, r_{i} \leqq t\right\}
$$

for $A \subset R^{n}$. For $h(r)=r^{\alpha}, \alpha>0$, this defines the usual $\alpha$-dimensional Hausdorff measure on all Borel sets in $R^{n}$ and

$$
\operatorname{dim}_{H} A=\inf \left\{\alpha>0: H_{h}^{*}(A)=0, h(r)=r^{\alpha}\right\}
$$

denotes the Hausdorff dimension of $A$.

2.5. Preliminary lemmas. The first two lemmas are well-known.

Suppose that $h$ is a measure function and that $\sigma$ is a finite measure in $R^{n}$ defined on all Borel sets. For $x \in R^{n}$ and $r>0$ write $\sigma(x, r)=\sigma\left(B^{n}(x, r)\right)$. 
2.6. Lemma. (Cf. [3, pp. 196-204].) If $\lambda>0$ and

$$
A_{\lambda}=\left\{x \in R^{n}: \sigma(x, r) \leqq h(r) / \lambda \text { for all } r>0\right\},
$$

then $H_{h}\left(R^{n} \backslash A_{\lambda}\right) \leqq c_{n} \lambda \sigma\left(R^{n}\right)$, where $c_{n}>0$ depends only on $n$.

2.7. Lemma. [7, Lemma 4] If $F:(0, \infty) \rightarrow R$ is decreasing, absolutely continuous on compact subintervals, and $\lim _{r \rightarrow \infty} F(r)=0, \lim _{r \rightarrow 0} F(r)=\infty$, then

$$
\int_{R^{n}} F(|x-y|) d \sigma(y)=-\int_{0}^{\infty} F^{\prime}(r) \sigma(x, r) d r .
$$

In order to estimate the upper $h$-measure density an interpolation lemma of type [7, Lemma 6] is needed:

2.8. Lemma. Suppose that $u \in L^{p}, p>1$, is non-negative and that $u\left[\complement B^{n}\left(r_{0}\right)=0\right.$. Then for all $\alpha>0$

$$
H_{h}\left(\left\{x \in R^{n}: v(x)>\Omega_{n}^{1-1 / p}\left(\frac{n-1}{\alpha} \int_{0}^{r_{0}} h(t)^{1 / p} t^{-n / p} d t+r_{0}^{1-n / p}\|u\|_{p}\right)\right\}\right) \leqq c_{n}\left(\alpha\|u\|_{p}\right)^{p},
$$

where

$$
v(x)=\int_{R^{n}} u(y)|x-y|^{1-n} d m(y)
$$

and $c_{n}$ is the constant of Lemma 2.6.

Proof. For $x \in R^{n}, r>0$, and non-negative measurable $w$ we let

By Hölder's inequality

$$
Q(w, x, r)=\int_{B^{n}(x, r)} w d m .
$$

$$
Q(u, x, r) \leqq \Omega_{n}^{1-1 / p} r^{n-n_{\prime}^{\prime} p} Q\left(u^{p}, x, r\right)^{1 / p} .
$$

On the other hand

$$
Q(u, x, r) \leqq \Omega_{n}^{1-1 / p} r_{0}^{n-n / p}\|u\|_{p}
$$

since $u\left[\mathbb{C} B^{n}\left(r_{0}\right)=0\right.$.

If $A \subset R^{n}$ is measurable we let

$$
\sigma(A)=\int_{A} u d m .
$$

Now $\sigma\left(R^{n}\right)<\infty$ since the support of $u$ is compact. Setting $F(r)=r^{1-n}, r>0$, Lemma 2.7 implies

$$
\begin{aligned}
v(x) & =\int_{R^{n}} u(y)|x-y|^{1-n} d m=\int_{R^{n}} F(|x-y|) d \sigma(y) \\
& =(n-1) \int_{0}^{\infty} Q(u, x, r) r^{-n} d r \\
& =(n-1) \int_{0}^{r_{0}} Q(u, x, r) r^{-n} d r+(n-1) \int_{r_{0}}^{\infty} Q(u, x, r) r^{-n} d r .
\end{aligned}
$$


Now by (2.9)

$$
\int_{0}^{r_{0}} Q(u, x, r) r^{-n} d r \leqq \Omega_{n}^{1-1 / p} \int_{0}^{r_{0}} Q\left(u^{p}, x, r\right)^{1 / p} r^{-n / p} d r
$$

and by (2.10)

$$
\begin{aligned}
\int_{r_{0}}^{\infty} Q(u, x, r) r^{-n} d r & \leqq \Omega_{n}^{1-1 / p} r_{0}^{n-n / p} \int_{r_{0}}^{\infty}\|u\|_{p} r^{-n} d r \\
& =\frac{\Omega_{n}^{1-1 / p}}{n-1} r_{0}^{1-n / p}\|u\|_{p} .
\end{aligned}
$$

Suppose that $\alpha>0$ and let

$$
B_{\alpha}=\left\{x \in R^{n}: Q\left(u^{p}, x, r\right) \leqq h(r) / \alpha^{p}\right\} .
$$

Define $\sigma(A)=\int_{A} u^{p} d m$ if $A \subset R^{n}$ is a Borel set and apply Lemma 2.6:

$$
H_{h}\left(R^{n} \backslash B_{\alpha}\right) \leqq c_{n} \alpha^{p} \sigma\left(R^{n}\right)=c_{n} \alpha^{p}\|u\|_{p}^{p} .
$$

If $x \in B_{\alpha}$, then by (2.12)

$$
\int_{0}^{r_{0}} Q(u, x, r) r^{-n} d r \leqq \Omega_{n}^{1-1 / p} \alpha^{-1} \int_{0}^{r_{0}} h(r)^{1 / p} r^{-n / p} d r
$$

and hence by (2.11), (2.13), and (2.14) for $x \in B_{\alpha}$

$$
v(x) \leqq K=\Omega_{n}^{1-1 / p}\left[\frac{n-1}{\alpha} \int_{0}^{r_{0}} h(r)^{1 / p} r^{-n / p} d r+r_{0}^{1-n / p}\|u\|_{p}\right] .
$$

This gives $\left\{x \in R^{n}: v(x)>K\right\} \subset R^{n} \backslash B_{\alpha}$ and the result follows.

\section{Upper bounds for measure densities}

Suppose that $C$ is a closed set in $R^{n}$ and $x \in R^{n}$. If $h$ is a measure function and $r>0$, then we let

$$
\Theta_{h}(x, C, r)=H_{h}\left(\bar{B}^{n}(x, r) \cap C\right) / h(r) .
$$

3.1. Theorem. If $p \in(1, n]$ and

$$
\int_{0}^{2 r} h(t)^{1 / p} t^{-n / p} d t \leqq A r^{(p-n) / p} h(r)^{1 / p}
$$

for some $A>0$ and all $r \in\left(0, r_{0}\right]$, then

$$
\Theta_{h}(x, C, r) \leqq c \operatorname{cap}_{p}(x, C, r), \quad r \in\left(0, r_{0}\right] .
$$

Here the constant $c$ depends only on $n, p$, and $A$. 
Proof. We may assume that $x=0$, and since $\Theta_{h}(0, C, r) \leqq 1$ for all $r>0$, we may also assume

$$
\operatorname{cap}_{p}(0, C, r)<K=\omega_{n-1}^{p} \Omega_{n}^{1-p} 2^{n-2 p}
$$

for all $r \in\left(0, r_{0}\right]$. Set

$$
I(r)=\int_{0}^{2 r} h(t)^{1 / p} t^{-n / p} d t .
$$

Let $\varepsilon>0$ and choose $w \in W\left(B^{n}(2 r), \bar{B}^{n}(r) \cap C\right)$ such that

$$
\operatorname{cap}_{p}\left(B^{n}(2 r), \bar{B}^{n}(r) \cap C\right) \geqq \int|\nabla w|^{p} d m-\varepsilon
$$

$$
\int|\nabla w|^{p} d m<K r^{n-p} .
$$

By (2.2)

$$
\begin{aligned}
w(x) & =\omega_{n-1}^{-1} \int|x-y|^{-n} \nabla w(y) \cdot(x-y) d m(y) \\
& \leqq \omega_{n-1}^{-1} \int|x-y|^{1-n}|\nabla w(y)| d m(y) .
\end{aligned}
$$

Now apply Lemma 2.8 with $u=|\nabla w| / \omega_{n-1}$ and $r_{0}=2 r$. The inequality (3.5) gives

$$
\Omega_{n}^{1-1 / p}(2 r)^{1-n / p}\|u\|_{p} \leqq \Omega_{n}^{1-1 / p}(2 r)^{1-n / p}\left(K r^{n-p}\right)^{1 / p} \omega_{n-1}^{-1}=1 / 2<1,
$$

hence we may choose $\alpha>0$ such that

Lemma 2.8 yields

$$
\Omega_{n}^{1-1 / p}\left[\frac{n-1}{\alpha} I(r)+(2 r)^{1-n / p}\|u\|_{p}\right]=1 .
$$

$$
\begin{gathered}
H_{h}\left(\bar{B}^{n}(r) \cap C\right) \leqq c_{n}\|u\|_{p}^{p}\left[\frac{(n-1) I(r)}{\Omega_{n}^{1 / p-1}-(2 r)^{1-n / p}\|u\|_{p}}\right]^{p} \\
\leqq \\
c_{n} 2^{p} \Omega_{n}^{p-1} \omega_{n-1}^{-p}(n-1)^{p} I(r)^{p}\left(\operatorname{cap}_{p}\left(B^{n}(2 r), \bar{B}^{n}(r) \cap C\right)+\varepsilon\right)
\end{gathered}
$$

where the inequality (3.5) has also been used. By the assumption (3.2), $I(r) \leqq$ $A r^{1-n / p} h(r)^{1 / p}$ and thus

$$
H_{h}\left(\bar{B}^{n}(r) \cap C\right) / h(r) \leqq c r^{p-n}\left(\operatorname{cap}_{p}\left(B^{n}(2 r), \bar{B}^{n}(r) \cap C\right)+\varepsilon\right)
$$

where $c=c_{n} 2^{n} \Omega_{n}^{p-1} \omega_{n-1}^{-p}(n-1)^{p} A^{p}$. Letting $\varepsilon \rightarrow 0$ gives the required result.

3.6. Corollary. Suppose that $h$ satisfies the condition (3.2). If $\operatorname{cap}_{p}(x, C)=0$, then $\Theta_{h}(x, C)=0$.

3.7. Corollary. If $1<p \leqq n$ and $\operatorname{cap}_{p}(x, C)=0$, then $\Theta_{h}(x, C)=0$ for $h(r)=r^{\alpha}$ and $\alpha>n-p$.

Proof. Let $h(r)=r^{\alpha}, \alpha>n-p$. In view of Corollary 3.6 it suffices to show that $h$ satisfies the condition (3.2). An easy calculation shows that this is true for all $r>0$ with $A=p(\alpha-n+p)^{-1} 2^{(\alpha-n+p) / p}$.

3.8. Theorem. Suppose that $C \subset R^{n}$ is closed and $1<p \leqq n$. If $\operatorname{cap}_{p}(x, C)=0$ for all $x \in C$, then $\operatorname{dim}_{H} C \leqq n-p$. 
Proof. If $\alpha>n-p$, then for $h(r)=r^{\alpha}$ Corollary 3.7 gives $\Theta_{h}(x, C)=0$ for all $x \in C$. By $[2,2.10 .19(2)], H_{h}^{*}(C)=0$. This shows $\operatorname{dim}_{H} C \leqq \alpha$ and the result follows.

3.9. Remarks. (a) It is well-known, see e.g. [6, p. 136] and [8, Corollary 2], that $\operatorname{cap}_{p} C=0$ implies $\operatorname{dim}_{H} C \leqq n-p$.

(b) Especially for $p=n$ it is interesting to know if the condition (3.2) would allow measure functions $h$ increasing more sharply at 0 than $h(r)=r^{\alpha}$ for any $\alpha>0$. Unfortunately, for $p \in(1, n]$ the condition (3.2) always implies that $h(r) \leqq c r^{\beta}$ for some $\beta>0$ and $c>0$ for all $r \in\left(0, r_{0}\right]$. To prove this choose an integer $i_{0} \geqq 2$ such that $2^{-i_{0}} \in\left(0, r_{0}\right]$. Then for $i \geqq i_{0}$

$$
\begin{gathered}
A h\left(2^{-i}\right)^{1 / p} 2^{i(n / p-1)} \geqq \int_{0}^{2^{-i+1}} h(t)^{1 / p} t^{-n / p} d t \\
\geqq \sum_{j=i}^{\infty} h\left(2^{-j}\right)^{1 / p} 2^{(j-1) n / p} 2^{-j}=\sum_{j=i}^{\infty} h\left(2^{-j}\right)^{1 / p} 2^{j(n / p-1)-n / p} .
\end{gathered}
$$

Assume first that $p \in(1, n)$. Fix $\beta>0$ and then an integer $k \geqq 2$ such that $i_{0} k>i_{0}+k$ and

$$
2^{\beta / p} A<2^{k(n / p-1)-n / p}
$$

Now for all $i \geqq i_{0}$

$$
h\left(2^{-i-k}\right) \leqq 2^{-\beta} h\left(2^{-i}\right)
$$

since otherwise

and thus

$$
h\left(2^{-i}\right) \geqq h\left(2^{-i-1}\right) \geqq \ldots \geqq h\left(2^{-i-k}\right)>2^{-\beta} h\left(2^{-i}\right)
$$

$$
\begin{gathered}
\sum_{j=i}^{\infty} h\left(2^{-j}\right)^{1 / p} 2^{j(n / p-1)-n / p}=2^{-\beta / p} h\left(2^{-i}\right)^{1 / p} \sum_{j=i}^{i+k} 2^{j(n / p-1)-n / p} \\
>2^{-\beta / p} h\left(2^{-i}\right)^{1 / p} 2^{(i+k)(n / p-1)-n / p} .
\end{gathered}
$$

But this combined with (3.10) and (3.11) gives a contradiction.

If $p=n$, fix $\beta>0$ and an integer $k \geqq 2$ so that $i_{0} k>i_{0}+k$ and $A<2^{-\beta / n-1} k$. Then it can be shown similarly that (3.12) holds.

To finish the proof let $r \in\left(0,2^{-i_{0} k}\right]$. Choose $i$ such that $r \in\left(2^{-i-1}, 2^{-i}\right]$ and then $m \geqq i_{0}$ so that $m k \leqq i<i+1 \leqq(m+1) k$. Since $i_{0} k \geqq i_{0}+k$ it follows from (3.12) by induction that

Hence

$$
h\left(2^{-j k}\right) \leqq 2^{-\beta\left(j-i_{0}+1\right)} h\left(2^{-i_{0}}\right), \quad j=i_{0}, i_{0}+1, \ldots
$$

$$
\begin{aligned}
h(r)^{k} & \leqq h\left(2^{-m k}\right)^{k} \leqq 2^{-\beta\left(m-i_{0}+1\right) k} h\left(2^{-i_{0}}\right)^{k} \\
& =2^{\beta i_{0} k} h\left(2^{-i_{0}}\right)^{k} 2^{-\beta(m+1) k} \leqq 2^{\beta i_{0} k} h\left(2^{-i_{0}}\right)^{k} r^{\beta}
\end{aligned}
$$

This gives the required result. 


\section{Lower bounds for measure densities}

Here we only consider measure functions $h$ of well-known type.

4.1. Theorem. Let

$$
\begin{aligned}
h(r) & =r^{n-p} \text { for } p \in(1, n), \quad r>0, \quad \text { and } \\
& =(\ln (1 / r))^{1-n} \text { for } p=n \text { and } 0<r<1 / 2 .
\end{aligned}
$$

If $C$ is a closed set in $R^{n}$, then

$$
\operatorname{cap}_{p}(x, C, r) \leqq c \Theta_{h}(x, C, r)
$$

for all $r>0$ if $p \in(1, n)$ and for $r \in(0,1 / 2)$ if $p=n$. The constant $c$ depends only on $n$ and $p$.

Proof. We may assume $x=0$. Consider first the case $1<p<n$. Fix $r>0$ and choose a covering $\bar{B}^{n}\left(x_{i}, r_{i}\right)$ of the set $\bar{B}^{n}(r) \cap C$ where $x_{i} \in \bar{B}^{n}(r)$. Assume $2 r_{i}<r$ for all $i$. Now by Lemma 2.4 and by (2.3)

$$
\begin{aligned}
\operatorname{cap}_{p}(0, C, r) & =r^{p-n} \operatorname{cap}_{p}\left(B^{n}(2 r), \bar{B}^{n}(r) \cap C\right) \\
& \leqq r^{p-n} \sum_{i=1}^{\infty} \operatorname{cap}_{p}\left(B^{n}\left(x_{i}, r\right), \bar{B}^{n}\left(x_{i}, r_{i}\right) \cap C\right) \\
& =c_{1} \sum_{i=1}^{\infty}\left[\left(r / r_{i}\right)^{q}-1\right]^{1-p} \leqq c_{1}\left(1-2^{-q}\right)^{1-p} \sum_{i=1}^{\infty}\left(r_{i} / r\right)^{n-p}
\end{aligned}
$$

where $c_{1}=\omega_{n-1} q^{p-1}, q=(n-p) /(p-1)$, and the inequality $2 r_{i}<r$ is used in the last step. Thus

$$
\operatorname{cap}_{p}(0, C, r) \leqq c \sum h\left(r_{i}\right) / h(r) .
$$

If $2 r_{i} \geqq r$ for some $i$, then, since $\operatorname{cap}_{p}(0, C, r) \leqq \omega_{n-1}\left(\left(1-2^{-q}\right) / q\right)^{1-p}$, the result is obvious.

In the case $p=n$ the estimate (4.2) can be written in the form

$$
\operatorname{cap}_{n}(0, C, r) \leqq c \sum\left[\frac{-\ln r_{i}}{-\ln r}\right]^{1-n}
$$

when the conditions $r<1 / 2$ and $2 r_{i}<r$ are used for the inequality $\left(\ln \left(r / r_{i}\right)\right)^{n-1} \geqq$ $c_{1}\left(-\ln r_{i}\right)^{n-1}(-\ln r)^{1-n}, c_{1}=(\ln 2)^{n-1} 2^{1-n}$ and $c=\omega_{n-1} c_{1}$. This yields the conclusion as above.

4.3. Corollary. Suppose $1<p \leqq n$ and let $h$ be as in Theorem 4.1. If $C \subset R^{n}$ is a closed set, then $\Theta_{h}(x, C)=0$ implies $\operatorname{cap}_{p}(x, C)=0$.

4.4. Remark. It is well-known, see e.g. [6, Theorem 7.2] or [10], that if $h$ is as in Theorem 4.1, then $H_{h}^{*}(C)<\infty$ gives $\operatorname{cap}_{p} C=0$. 
4.5. Corollary. If $C$ is a closed set in $R^{n}$ and $\operatorname{cap}_{p}(x, C)=0$ for all $x \in C$, then $\operatorname{cap}_{q} C=0$ for $q \in(1, p)$.

Proof. By Theorem $3.8 \operatorname{dim}_{H} C \leqq n-p$. Consequently $H_{h}^{*}(C)=0$ for $h(r)=r^{\alpha}$, $\alpha>n-p$. By Remark $4.4 \operatorname{cap}_{q} C=0$ for $q \in(1, p)$.

4.6. Corollary. Suppose that $1<p \leqq n$. If $C$ is a closed set in $R^{n}$ such that $\operatorname{cap}_{p} C>0$ and $\operatorname{cap}_{p}(x, C)=0$ for all $x \in C$, then $\operatorname{dim}_{H} C=n-p$. Moreover, $H_{h}^{*}(C)=\infty, h(r)=r^{n-p}$.

Proof. The case $p=n$ has been handled by Theorem 3.8. If $\alpha=n-p>0$ and if $H_{h}^{*}(C)<\infty, h(r)=r^{\alpha}$, then by Remark $4.4 \operatorname{cap}_{p} C=0$. Consequently, $\operatorname{dim}_{H} C \geqq$ $n-p$ and the opposite inequality follows from Theorem 3.8.

4.7. Example. Here we construct for $p=n$ a compact set $C \subset R^{n}$ such that $\operatorname{cap}_{n} C>0$ but $\operatorname{cap}_{n}(x, C)=0$ for all $x \in R^{n}$. In fact we shall show that even the condition $M(x, C)<\infty$ for all $x \in C$ holds. The condition $M(x, C)<\infty$, cf. [4] and [5], means that there exists a non-degenerate continuum $K \subset[C \cup\{x\}$ such that $x \in K$ and the $n$-modulus of the curve family joining $K$ and $C$ is finite. By [5, Theorem 3.1] $M(x, C)<\infty$ implies $\operatorname{cap}_{n}(x, C)=0$. A set of this type is of function theoretic interest, see [11].

To this end let $k \in(1,2)$ and define $l_{i}^{\prime}=\exp \left(-k^{n i /(n-1)}\right), i=0,1, \ldots$ Fix $i_{0}$ such that $4 \sqrt{n} l_{i+1}^{\prime}<l_{i}^{\prime}$ for $i \geqq i_{0}$ and write $l_{i}=l_{i+i_{0}}^{\prime}, i=0,1, \ldots$. Let $\Delta_{0}$ be a closed interval of length $l_{0}$ and set $E_{0}=\Delta_{0} \times \ldots \times \Delta_{0}$ ( $n$ times). Denote by $F_{1}$ the union of two closed intervals $\Delta_{1}^{1}$ and $\Delta_{1}^{2}$ of length $l_{1}$ lying in $\Delta_{0}$ and containing both ends of $\Delta_{0}$. Set $E_{1}=F_{1} \times \ldots \times F_{1}$ and carry out the same operations in the intervals $\Delta_{1}^{1}$ and $\Delta_{1}^{2}$ using $l_{2}$ instead of $l_{1}$. Four intervals $\Delta_{2}^{i}, i=1,2,3,4$, are obtained. Let their union be $F_{2}$ and set $E_{2}=F_{2} \times \ldots \times F_{2}$. This process can be continued and define $C=\bigcap_{i=0}^{\infty} E_{i}$. Each set $E_{i}$ consists of $2^{\text {in }}$ closed cubes $Q_{i}^{j}, j=1, \ldots, 2^{i n}$, with sides of length $l_{i}$.

The set $C$ is of positive $n$-capacity since

$$
\sum_{i=1}^{\infty} 2^{n i /(1-n)} \ln \left(l_{i} / l_{i+1}\right)<\infty,
$$

cf. [6, Theorem 7.4 and the following Remark]. For relations between the capacity used in [6] and the variational capacity used in this paper see [8, Theorems 6.1 and 6.2].

Next we consider the condition $M\left(x_{0}, C\right)<\infty$. Fix $x_{0} \in C$. For each $i \geqq 1$ choose a cube $Q_{i}$ in the collection $\left\{Q_{i}^{j}\right\}$ such that $x_{0} \in Q_{i}$. Now it is easy to construct a continuum $K_{i+1} \subset Q_{i}$ consisting of line segments $L_{1}, L_{2}, L_{3}$ in the plane $T=\left\{x \in R^{n}: x_{j}=\left(x_{0}\right)_{j}, j=3, \ldots, n\right\}$ and such that $L_{1}$ joins the midpoint of a face of $T \cap Q_{i}$ to the center of $T \cap Q_{i}, L_{2}$ is a part of a similar segment and $L_{3}$ is perpendicular to $L_{2}$ and joins the midpoint of a face of $T \cap Q_{i+1}$ to the endpoint of $L_{2}$. Now $d\left(K_{i+1}, Q_{i+1}^{\prime} \backslash Q_{i+1}\right) \geqq l_{i} / 4$ and $d\left(K_{i+1}, Q_{i+2}^{\prime}\right) \geqq l_{i+1} / 4$ where $Q_{k}^{\prime}=Q_{k-1} \cap \bigcup_{j} Q_{k}^{j}$. 
Set $K=\bigcup_{i=1}^{\infty} K_{i+1} \cup\left\{x_{0}\right\}$. Then after a suitable selection of the continua $K_{i+1}, K$ is a non-degenerate continuum with $x_{0} \in K$.

If $E$ and $F$ are closed sets in $R^{n}$. we denote by $\Delta(E, F)$ the family of all paths joining these sets in $R^{n}$. For properties of the $n$-modulus $M(\Delta(E, F))$ of the path family $\Delta(E, F)$ we refer to [9].

It remains to show $M(\Delta(K, C))<\infty$. By [9, Theorem 6.2] for each $i \geqq 1$

$$
\begin{aligned}
M\left(\Delta\left(K_{i+1}, C\right)\right) & \leqq M\left(\Delta\left(K_{i+1}, Q_{i+2}^{\prime}\right)\right)+M\left(\Delta\left(K_{i+1}, \bigcup_{j=1}^{i+1}\left(Q_{j}^{\prime} \backslash Q_{j}\right)\right)\right) \\
& \leqq M\left(\Delta\left(K_{i+1}, Q_{i+2}^{\prime}\right)\right)+\sum_{j=1}^{i+1} M\left(\Delta\left(K_{i+1}, Q_{j}^{\prime} \backslash Q_{j}\right)\right)
\end{aligned}
$$

and we estimate each term separately.

Fix $1 \leqq j \leqq i$. Now $K_{i+1} \subset Q_{i}$ and $\left(Q_{j}^{\prime} \backslash Q_{j}\right) \cap B^{n}\left(x_{0}, l_{j-1} / 2\right)=\emptyset$, thus

$$
\begin{gathered}
M\left(\Delta\left(K_{i+1}, Q_{j}^{\prime} \backslash Q_{j}\right)\right) \leqq \omega_{n-1}\left(\ln \left[\left(l_{j-1} / 2\right) /\left(l_{i} \sqrt{n} / 2\right)\right]\right)^{1-n} \\
=\omega_{n-1} k^{-n\left(i_{0}+i\right)}\left[1-k^{n\left(i_{0}+i\right) /(1-n)} \ln \sqrt{n}-k^{n(j-1-i) /(n-1)}\right]^{1-n} \leqq c_{1} k^{-n i}
\end{gathered}
$$

where $c_{1}$ depends only on $n, k$, and $i_{0}$.

If $j=i+1$, then because of the quasi-invariance of the $n$-modulus under biLipschitz mappings, see [9], it is easy to see that there is $c_{2}^{\prime}>0$ depending only on $n$ such that

$$
\begin{aligned}
M\left(\Delta\left(K_{i+1}, Q_{i+1}^{\prime} \backslash Q_{i+1}\right)\right) & \leqq c_{2}^{\prime} M\left(\Delta\left(\bar{B}^{n}\left(l_{i+1}\right), S^{n-1}\left(l_{i}\right)\right)\right) \\
& =c_{2}^{\prime} \omega_{n-1}\left(\ln \left(l_{i} / l_{i+1}\right)\right)^{1-n} \leqq c_{2} k^{-n i}
\end{aligned}
$$

and $c_{2}$ depends on the same constants as $c_{1}$.

As above the estimate

$$
M\left(\Delta\left(K_{i+1}, Q_{i+2}^{\prime}\right)\right) \leqq c_{3}^{\prime} M\left(\Delta\left(\bar{B}^{n}\left(l_{i+2}\right), S^{n-1}\left(l_{i+1}\right)\right)\right) \leqq c_{3} k^{-n(i+1)}
$$

is obtained where $c_{3}$ depends on the same constants as $c_{1}$.

Finally, the inequalities (4.8)-(4.11) yield

$$
\begin{aligned}
M(\Delta(K, C)) & \leqq \sum_{i=1}^{\infty} M\left(\Delta\left(K_{i+1}, C\right)\right) \\
& \leqq \sum_{i=1}^{\infty}\left[c_{3} k^{-n(i+1)}+c_{2} k^{-n i}+(i+1) c_{1} k^{-n i}\right] \\
& \leqq\left(c_{1}+c_{2}+c_{3}\right) \sum_{i=1}^{\infty}(i+1) k^{-n i}<\infty
\end{aligned}
$$

This shows that $M\left(x_{0}, C\right)<\infty$. 


\title{
References
}

[1] Carleson, L.: Selected problems on exceptional sets. - Van Nostrand Mathematical Studies 13, D. Van Nostrand Company, Inc., Princeton, N. J., Toronto-London-Melbourne, 1967.

[2] Federer, H.: Geometric measure theory. - Springer-Verlag, Berlin-Heidelberg-New York, 1969.

[3] LANdKof, N. S.: Foundations of modern potential theory. - Springer-Verlag, Berlin-Heidelberg-New York, 1972.

[4] Martio, O.: Equicontinuity theorem with an application to variational integrals. - Duke Math. J. 42, 1975, no. 3, 569-581.

[5] Martio, O., and J. Sarvas: Density conditions in the $n$-capacity. - Indiana Univ. Math. J. 26, 1977, no. 4, 761-776.

[6] Maz'JA, V. G., and V. P. Havin: A nonlinear potential theory. - Uspehi Mat. Nauk 27, 1972, no. 6 (168), 67-138 (Russian).

[7] RešEtnJAK, Ju. G.: Space mappings with bounded distortion. - Sibirsk. Mat. Ž. 8, 1962 , 629-658 (Russian).

[8] REŠETNJAK, JU. G.: The concept of capacity in the theory of functions with generalized derivatives. - Ibid. 10, 1969, 1109-1138 (Russian).

[9] VÄISÄLÄ, J.: Lectures on $n$-dimensional quasiconformal mappings. - Lecture Notes in Mathematics 229, Springer-Verlag, Berlin-Heidelberg-New York, 1971.

[10] VÄISÄLÄ, J.: Capacity and measure. - Michigan Math. J. 22, 1975, 1-3.

[11] Vuorinen, M.: Exceptional sets and boundary behavior of quasiregular mappings in $n$-space. Ann. Acad. Sci. Fenn. Ser. A I Math. Dissertationes 11, 1976, 1-44.

\author{
University of Helsinki \\ Department of Mathematics \\ SF-00100 Helsinki 10 \\ Finland
}

Received 13 March 1978 